\title{
Mapping of polarization and detrapping effects in synthetic single crystal chemical vapor deposited diamond by ion beam induced charge imaging
}

\author{
A. Lohstroh, ${ }^{\text {a) }}$ P. J. Sellin, S. G. Wang, A. W. Davies, and J. M. Parkin \\ Department of Physics, University of Surrey, Guildford GU2 7XH, United Kingdom
}

(Received 6 October 2006; accepted 30 December 2006; published online 28 March 2007)

\begin{abstract}
Diamond has been regarded as a promising radiation detector material for use as a solid state ionizing chamber for decades. The parameters degrading the charge transport from what is expected from an ideal crystal are still not completely understood. Recently, synthetic chemical vapor deposited (CVD) single crystal diamond has become available, offering the opportunity to study the properties of synthesized material independent of grain boundaries. We have studied the charge transport of a synthetic single crystal diamond with $\alpha$-particle induced charge transients as a function of temperature and established the presence of a shallow hole trap with an activation energy of $0.29 \pm 0.02 \mathrm{eV}$ in some parts of the detector. Ion beam induced charge imaging has been used to study the spatial variations of the charge transport in a synthetic single crystal diamond. Pulses influenced by the shallow hole trap had their origin close to the substrate/CVD interface of the sample. They could be clearly distinguished from pulses affected by reduced charge carrier velocities due to polarization phenomena, which varied systematically with the growth direction of the CVD diamond material. (C) 2007 American Institute of Physics. [DOI: 10.1063/1.2653669]
\end{abstract}

\section{INTRODUCTION}

Diamond is a unique material combining a number of extreme intrinsic properties, which make it an interesting device material for radiation detection, ranging from ultraviolet and $\mathrm{x}$-ray detection to $\alpha-, \beta-, \gamma$-particle and neutron monitoring. ${ }^{1,2}$ The wide band gap of $5.5 \mathrm{eV}$ means that an ideal diamond is solar blind and has an extremely low number of free charge carriers excited thermally from the valence band into the conduction band, which translates into very low detector leakage currents. ${ }^{3}$ The material's radiation hardness and its chemical inertness are beneficial for long term operation in harsh environments either in terms of strong radiation fields ${ }^{4}$ or in aggressive ambient conditions. For example, no significant changes in the signal amplitude due to radiation damage by high energy protons in polycrystalline diamond have been found for doses up to $10^{15}$ protons $\mathrm{cm}^{-2}{ }^{5}$

The low atomic number of carbon is considered as tissue equivalent and has triggered a long history of research in diamond-based dosimetry. ${ }^{6}$ The large charge carrier mobilities and saturation velocities will allow very fast signal current pulses. The charge transport properties crucially determine the signal amplitude and reproducibility, thus the performance of the detector. Consequently the performance varies significantly with the defect distribution within the material and polarization and priming phenomena are frequently observed in natural and synthetic diamonds. ${ }^{2,7}$

Synthetic diamond has been purely polycrystalline material for many years and its spectroscopic application has been limited by strong charge carrier trapping at the defect rich grain boundaries. ${ }^{8,9}$ On the other hand, the defects intro-

${ }^{a)}$ Electronic mail: a.lohstroh@surrey.ac.uk duced during the synthesis of natural gem stones cannot be controlled at all and only a very small percentage of selected natural diamonds are suitable for detector applications. ${ }^{2} \mathrm{Al}-$ though the high temperature/high pressure (HTHP) production of single crystal diamond is possible, its drawbacks are the extreme synthesis conditions as well as the difficulty to scale the process to larger areas. ${ }^{10}$ Additionally, it is often hard to achieve the purity required for electronic applications, including radiation detection. In recent years, very promising high purity synthetic single crystal diamonds of more than $100 \mu \mathrm{m}$ thickness grown by microwave plasma chemical vapor deposition (CVD) have become available. ${ }^{11-13}$ They offer the opportunity to study the charge transport of this engineered material independent of grain boundaries.

Ion beam induced charge (IBIC) imaging is a useful tool to image the spatial variations in charge transport and has been applied in numerous studies of diamond detector performance. ${ }^{14-16}$

In this work, we have evaluated the charge transport through a piece of synthetic CVD single crystal diamond using $5.48 \mathrm{MeV} \alpha$-particle induced charge transient pulses between 200 and $300 \mathrm{~K}$ at applied field strength between 300 and $4000 \mathrm{~V} \mathrm{~cm}^{-1}$. The temperature dependence of the transient charge pulses establishes the presence of a shallow hole trap level previously reported in the literature. ${ }^{17,18}$ IBIC imaging with $2.6 \mathrm{MeV}$ protons has been performed at 296 and $270 \mathrm{~K}$. Detector areas of reduced charge carrier velocities due to polarization effects are distinguished in these images from areas of delayed charge transport due to the re-emission of charge carriers out of the aforementioned shallow hole traps. 


\section{SIGNAL ANALYSIS}

Ionizing radiation interacting with the material of a semiconductor device will excite electrons from the valence band into the conduction band, which drift with a velocity $v=\mu E$ according to the electric field $E$ throughout the detector and their mobility $\mu$, thereby inducing a current in the outside circuit. The number of created free charge carriers $Q_{0}$ is proportional to the energy absorbed by the detector. The integrated current, i.e., the charge signal, is proportional to the drift length of the carriers. In the special case of a detector with a sandwich electrode structure with an electrode distance $d$ and charge carrier creation at a depth $x$ close to one electrode $(x \ll d)$, mainly one charge carrier type (either electrons or holes) will contribute to the signal. If the electric field throughout the detector is constant and no re-emission of charge carriers out of shallow trap levels occurs, then the current pulse $I(t)$ starts when the radiation interaction takes place at $t=0$. The current drops exponentially with a time constant given by the carrier lifetime $\tau$ until the carriers reach the opposite electrode after the transit time $T_{R}$ as given in Eq. (1).

$$
\begin{aligned}
& I(t) \sim \exp \left(-\frac{t}{\tau}\right) \\
& \Rightarrow Q(t)=\int_{0}^{t} I\left(t^{\prime}\right)^{d t^{\prime}}=\frac{\tau}{T_{R}} \frac{d-x}{d}[1-\exp ( \\
&\left.\left.-\frac{t}{\tau}\right)\right] \quad \text { for } t<T_{R} .
\end{aligned}
$$

Reported charge carrier mobilities in diamond are larger than $1000 \mathrm{~cm}^{2} \mathrm{~V}^{-1} \mathrm{~s}^{-1}$. 3,19,20 Assuming a typical device with a maximum thickness $d$ of $0.5 \mathrm{~mm}$ and an electric field strength of more than $1 \mathrm{kV} \mathrm{cm}^{-1}$, it follows that the transit time can be expected to be less than $50 \mathrm{~ns}$.

The ratio of the total induced charge $Q_{\text {ind }}$ [where $Q_{\text {ind }}$ $=Q(t \rightarrow \infty)$, i.e., the time integrated current signal] over the created charge is defined as the charge collection efficiency (CCE), which is given by the Hecht equation (2), ${ }^{21}$

$$
\frac{Q_{\text {ind }}}{Q_{0}}=\mathrm{CCE}=\frac{\mu \tau E}{d}\left[1-\exp \left(\frac{x-d}{\mu \tau E}\right)\right] \text {. }
$$

The trapping of drifting charge carriers at deep defect levels causes the buildup of an internal space charge inside the material of the device. ${ }^{22,23}$ Subsequently created electron hole pairs will experience a weakened electric field, which causes a reduction in velocity and consequently in CCE. This process is referred to as polarization. On the other hand, priming, sometimes also called pumping, describes the increase in charge carrier lifetime due to the filling of defect levels with trapped charge carriers. Priming with electron, $\mathrm{x}$ or $\gamma$-ray pre-irradiation can be used to improve detector performance. $^{24}$

Delayed hole transport in diamond at $t>T_{R}$ has been observed in primed and unprimed, single and polycrystalline diamond detectors, which is caused by the re-emission of holes out of a shallow trap with detrapping time constants $\tau_{D}$ in the order of a few microseconds, clearly longer than $T_{R}$.

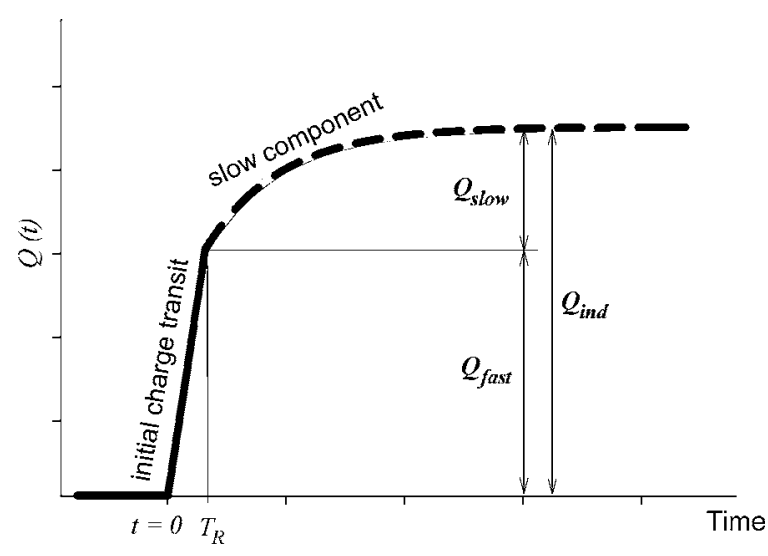

FIG. 1. Schematic of an induced charge pulse with a slow component.

They contribute to the charge signal, causing a "slow" component visible in the integrated signal current pulse, as illustrated in Fig. 1. ${ }^{17,18}$ If charge carriers are only trapped once in a shallow trap during their transit, then the time constant of the slow component will be independent of the electric field and the amplitude $Q(t)$ of the integrated current pulse can be described by Eq. (3) for $t>T_{R}$, where $Q_{\text {slow }}$ and $Q_{\text {fast }}$ are the amplitude of the slow and fast component, respectively, so that $Q_{\text {ind }}=Q_{\text {fast }}+Q_{\text {slow }}$.

$$
Q(t)=Q_{\text {fast }}+Q_{\text {slow }}\left[1-\exp \left(-\frac{t}{\tau_{D}}\right)\right] \text {. }
$$

The detrapping time constant $\tau_{D}$ can be extracted from Eq. (4) using a standard linear regression, as demonstrated by Balducci et $a$. $^{25}$

$$
\ln \left[Q_{\text {ind }}-Q(t)\right]=\ln Q_{\text {slow }}-\frac{t}{\tau_{D}} .
$$

For a purely thermally activated detrapping process, the relationship between $\tau_{D}$ and the temperature $T$ is given by Eq. (5) which is dominated by the activation energy $E_{A} ; c$ is a trap specific constant. ${ }^{26}$

$$
\tau_{D}=c T^{2} \exp \left(-\frac{E_{A}}{k_{B} T}\right) .
$$

\section{EXPERIMENTAL DETAILS}

The sample studied in this work has been grown by CVD on a single crystalline HPHT synthesized diamond substrate by Element Six Ltd. The growth process is similar to the one described by Isberg et al. ${ }^{11}$ Additional nitrogen has been deliberately introduced into the growth reactor at several stages of the growth process, which results in thin nitrogen rich layers at varying distances from the substrate material. The sample has been cut vertically to the growth direction, with dimensions of $2.5 \times 2.9 \times 0.49 \mathrm{~mm}^{3}$. Prior to the contacting, a DiamondView image, was acquired using an instrument made by the Diamond Trading Company Ltd. $^{27}$ The instrument uses the short wave $(<227 \mathrm{~nm}$ wavelength) ultraviolet emission from xenon lamps to excite near surface luminescence from diamond samples. Planar contacts 


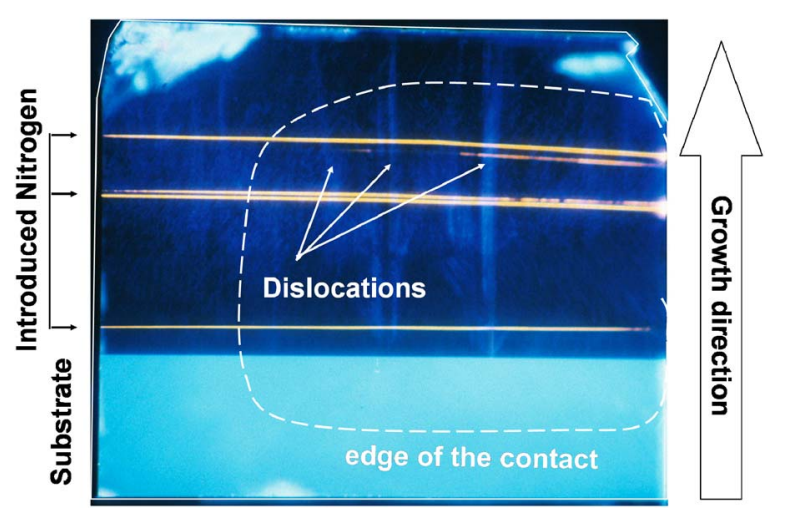

FIG. 2. (Color online) Photoluminescence image (Courtesy of Element Six Ltd.).

of $50 \mathrm{~nm}$ titanium followed by $300 \mathrm{~nm}$ gold were evaporated on the sample surface and annealed at $400{ }^{\circ} \mathrm{C}$ to achieve Ohmic contact properties. ${ }^{28}$

Charge pulses were induced by a non-collimated ${ }^{241} \mathrm{Am}$ $5.48 \mathrm{MeV} \alpha$-particle source in vacuum at pressures below $10^{-1}$ mbar, with sample temperatures between 200 and $300 \mathrm{~K}$ and typical registered event rates of less than $20 \mathrm{~s}^{-1}$. Spatially resolved IBIC imaging has been performed with 2.6 MeV protons at the Surrey Microbeam line at 296 and $270 \mathrm{~K}^{29}$ The beam dimensions were less than $4 \times 4 \mu \mathrm{m}^{2}$ and the incident number of protons was typically between 500 and $1000 \mathrm{~s}^{-1}$, which results in an estimated maximum accumulated dose of $10^{10}$ particles $\mathrm{cm}^{-2}$ for all the experiments performed.

During the irradiation, the bias has been applied at the irradiated contact via an Ortec 142 A charge sensitive preamplifier. The signal has been passed on to an Ortec 570 shaping amplifier followed by a multichannel analyzer for the pulse height spectra acquisition. The amplitudes have been calibrated in terms of CCE using a pulser, cross correlated with a $100 \%$ efficient silicon pin diode, assuming the average energy needed to create an electron hole pair of 3.6 and $13.2 \mathrm{eV}$ in silicon ${ }^{21}$ and diamond, ${ }^{30}$ respectively. The ranges $x$ of the $5.48 \mathrm{MeV} \alpha$-particles and $2.6 \mathrm{MeV}$ protons are 15 and $35 \mu \mathrm{m}$, respectively, as calculated by SRIM. ${ }^{31}$ This is small compared to the sample thickness $d=490 \mu \mathrm{m}$. Therefore, the charge induced under negative bias is mainly due to electron drift, whereas charge induced under positive bias is sensitive to hole transport.

Transient charge pulses have been acquired using a digital oscilloscope with a maximum sampling rate of $2.5 \mathrm{Gs} / \mathrm{s}$ in combination with the non-collimated $\alpha$-particle source, as described by Wang et al. ${ }^{32}$ The time resolution of that system is limited by the system time response function dominated by the preamplifier rise time and results in $\sim 50 \mathrm{~ns}$. The time resolved ion beam imaging was performed by the digital IBIC setup described by Sellin et al. ${ }^{33}$ The minimum rise time of that setup was about $80 \mathrm{~ns}$ during these experiments.

\section{IV. $\alpha$-PARTICLE SPECTROSCOPY}

Figure 2 shows the luminescence image of the sample. The bright area at the bottom is the HPHT substrate material. The horizontal luminescent lines are the introduced nitrogen
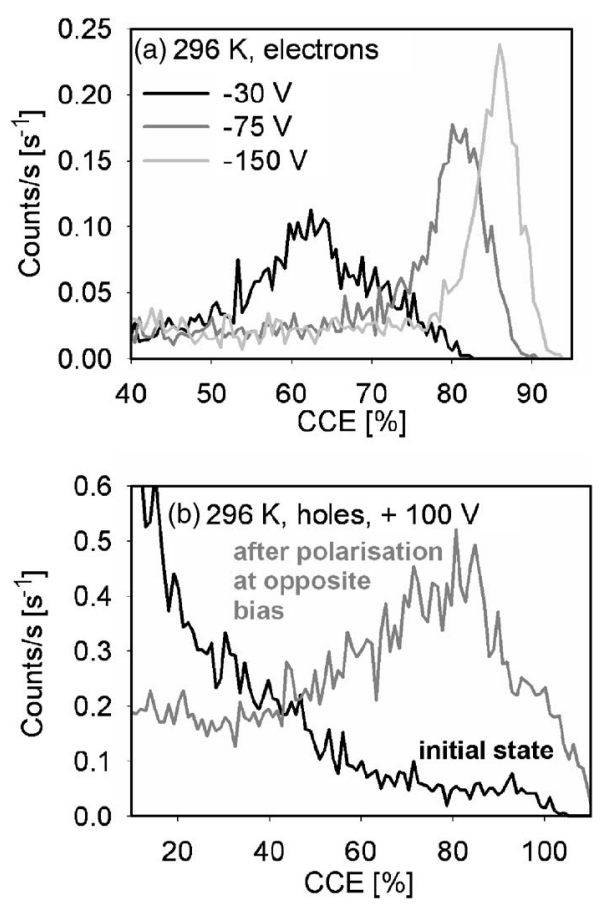

FIG. 3. Pulse height distribution acquired during $5.48 \mathrm{MeV} \alpha$-particle irradiation.

rich layers. A cathodoluminescence experiment ${ }^{34}$ has shown that these nitrogen rich layers emit a signal with a zero phonon line at $2.15 \mathrm{eV}$, originating from a neutral nitrogen vacancy complex. ${ }^{35}$ Additionally, vertical line structures with enhanced blue band $A$ luminescence are also distinguishable. They are probably caused by dislocations or dislocation bundles. $^{36}$

We acquired $\alpha$-particle induced charge amplitude spectra and rise time distributions as a function of bias voltage between 200 and $300 \mathrm{~K}$, some examples are displayed in Fig. 3. The induced amplitudes did not change significantly with temperature. Electron sensitive CCE distributions show well resolved peaks, as displayed in Fig. 3(a). In contrast, the hole sensitive data reflect poor hole transport. The hole spectra were very sensitive to polarization effects and improved after irradiation at negative bias voltage due to the buildup of an internal electric field, which is illustrated by the two graphs in Fig. 3(b).

We have acquired the $10 \%-90 \%$ rise time $\tau_{10 \%-90 \%}$ under decreasing negative bias from -100 to $-15 \mathrm{~V}$ between 200 and $300 \mathrm{~K}$. The observed $\tau_{10 \%-90 \%}$ increased from 50 to $70 \mathrm{~ns}$ as a function of bias voltage. Due to the intrinsic rise time of the system of $50 \mathrm{~ns}$, the combination of $\tau_{10 \%-90 \%}$ and CCE data allows only a rough estimate of the lifetime using Eq. (1), which is in the order of 15-30 ns. Assuming typical mobility values reported in the recent literature of more than $1000 \mathrm{~cm}^{2} \mathrm{~V}^{-1} \mathrm{~s}^{-1}, 3,19,20$ it is expected that the transit time $T_{R}$ will be shorter than the time resolution of our system at bias voltages larger than $\pm 50 \mathrm{~V}$ for both electrons and holes, which is in agreement with our observation.

The lower amplitudes of the hole signals under the low intensity $\alpha$-particle irradiation made a similar analysis of the hole transit time impossible. Nevertheless, a small percentage of the hole pulses acquired between +100 and $+200 \mathrm{~V}$ 

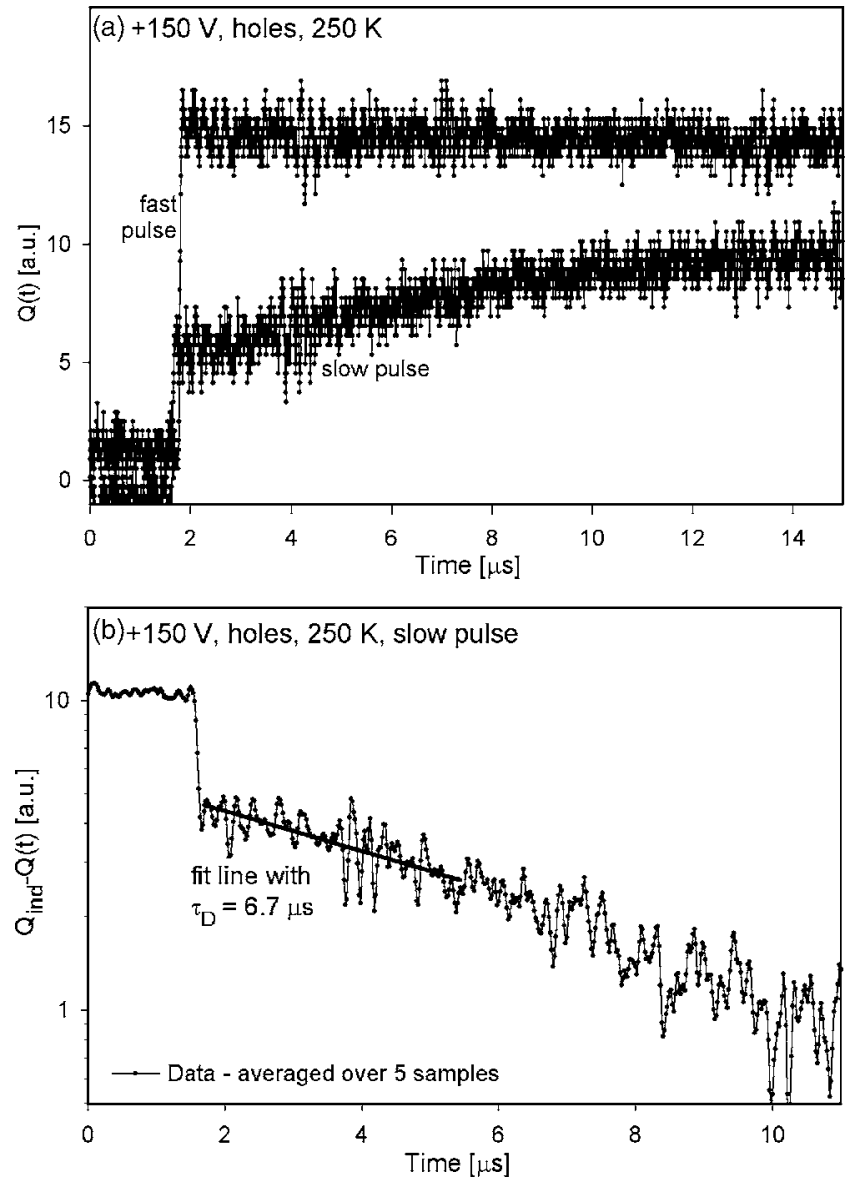

FIG. 4. (a) Comparison between a fast pulse and a slow pulse acquired within the same data set. (b) Fit according to Eq. (4) of the slow pulse shown in (a).

exhibited the characteristic temperature dependent slow component due to re-emission of charge carriers out of shallow hole traps, as described in various articles. ${ }^{17,18}$ An example wave form for each case-with and without a slow component acquired in the same experimental conditions-is shown in Fig. 4(a). We have fitted a detrapping time to each pulse exhibiting a slow component using Eq. (4). The data for the fit have been averaged over five consecutive sample points to reduce the noise in the wave form. Only pulses with a sufficiently large $Q_{\text {slow }}(>2.6 \%$ CCE) have been considered to reduce the influence of misleading fits of wave forms, which actually do not have a slow component. The fit interval begins at $t=60 \mathrm{~ns}$ and was limited to the first $33 \%$ of the rising pulse length, because the impact of noise frequently distorts the fit result when $Q(t)$ approaches $Q_{\text {ind }}$. Finally, fits which described the data very poorly $\left(r^{2}<0.4\right)$ were rejected. Figure 4(b) illustrates the procedure by showing the fit to the slow pulse shown in the Fig. 4(a).

The pulse by pulse fitting of pulses results in distributions of detrapping times extracted from pulses exhibiting a slow component acquired at the same temperature and voltage setting, as exemplified in the inset of Fig. 5. These distributions have resolvable peaks between 240 and $296 \mathrm{~K}$, and the peak positions were used to produce an Arrhenius plot following Eq. (5), as shown in Fig. 5. It illustrates that there is hardly any variation in detrapping time with voltage.

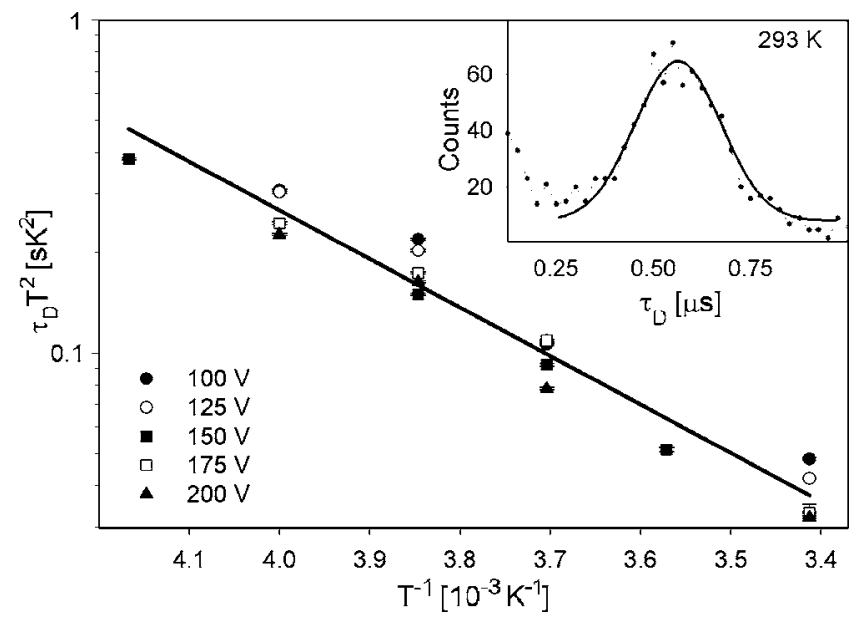

FIG. 5. Arrhenius plot extracted from the detrapping time distributions at various temperatures and voltages. Inset: Example histogram of a detrapping time distribution.

The absence of electric field dependence indicates that multiple trapping-detrapping effects are negligible. The graph yields an activation energy of the trap of $0.29 \pm 0.02 \mathrm{eV}$. A defect with similar time characteristics has been found previously in single ${ }^{37}$ and polycrystalline diamond ${ }^{17,18}$ and is in agreement with the activation energy of a boron related defect identified by photon and electron beam induced current transient studies. ${ }^{38,39}$

\section{ION BEAM INDUCED CHARGE (IBIC) IMAGING}

Figure 6 displays the CCE distribution extracted from the IBIC experiment at $296 \mathrm{~K}$ at $+100 \mathrm{~V}$. The image is a qualitative representative for all images acquired at applied field strength larger than $2 \mathrm{kV} \mathrm{cm}^{-1}$, positive and negative. Similar images were also acquired at $\pm 100 \mathrm{~V}$ at $270 \mathrm{~K}$, confirming that the CCE variation with temperature is negligible in this range. It shows that the CCE of the HTHP substrate material is below the threshold level of the data acquisition system and the lines associated with the nitrogen vacancy luminescence have a strongly reduced CCE. The vertical

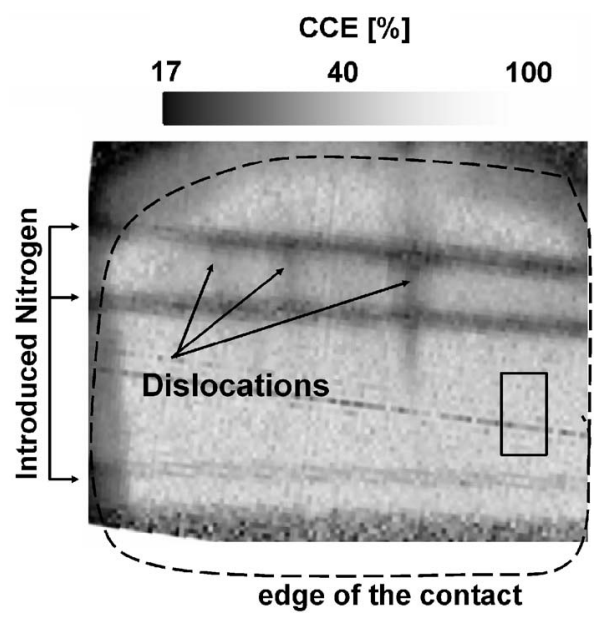

FIG. 6. Hole sensitive CCE image acquired at $+100 \mathrm{~V}$ with $2.6 \mathrm{MeV}$ protons at $296 \mathrm{~K}$, the pulse height spectra of the rectangular area are presented in Fig. 7. 


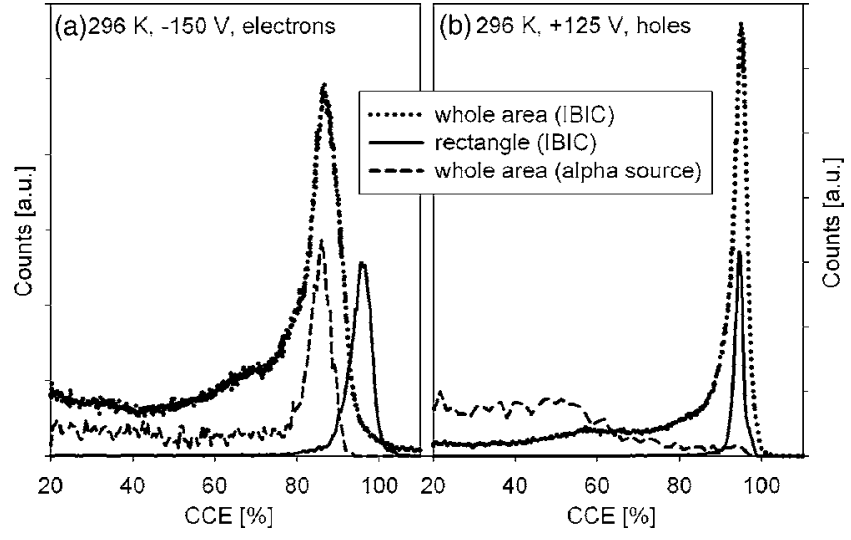

FIG. 7. Comparison of pulse height distributions for (a) electrons and (b) holes.

lines with enhanced band $A$ luminescence also exhibit a slightly reduced CCE compared to their surroundings with lower band $A$ intensity. This is consistent with nitrogen and dislocations acting as recombination centers, ${ }^{40}$ reducing the lifetime and hence the CCE, as it has been reported and discussed previously. ${ }^{34}$

Figure 7 gives a comparison of the IBIC spectra acquired from the whole scan area compared to a selected high quality region of the device. In addition, the performance obtained with the non-collimated $\alpha$ source irradiating the whole detector area is also displayed. Figure 7(a) demonstrates that the electron signals acquired during the ion beam irradiations are comparable to the ones acquired with the $\alpha$ source. Thus the electron transport is hardly dependent on the ion species, interaction depth, and incident dose rate. CCEs above $90 \%$ are achieved over a wide area of the device. In contrast to that, in a hole sensitive mode, the detector shows a greatly improved performance in the ion beam compared to the $\alpha$ irradiation. In the literature, an increase of signal height with increasing depth range of the incident radiation has been suggested, due to enhanced trapping at a highly defective contact interface layer. ${ }^{41}$ This is unlikely to be the reason in our case, as electron transport does not show the same effect. The improved hole CCE observed during the proton beam irradiation is most likely caused by the approximately 50 times larger incident particle rate of the ion beam. Created charge carriers fill the deep trap levels within the bulk material, which stay occupied by the trapped charge over long time scales. The trapping probability for subsequently created charge carriers reduces and their lifetime and the CCE increase. High CCE centroids, as shown in Fig. 7(b), were only observed in the IBIC experiments, where the effect of priming due to irradiation is much stronger than during the $\alpha$-experiments, due to the larger incident dose rate on to the device. It can be assumed that priming is responsible for the deactivation of bulk hole traps, ${ }^{42,43}$ which explains the stronger effect on hole transport than on the electron performance.

The CCE images acquired at very low voltages shown in Fig. 8 seem to complement each other, i.e., areas with low electron CCE have a large hole CCE and vice versa. This effect is at least partly caused by the buildup of polarization

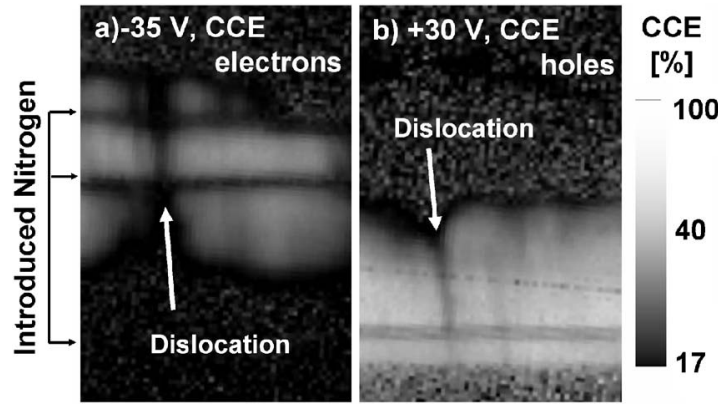

FIG. 8. CCE images acquired at $296 \mathrm{~K}$ : (a) electron sensitive at $-35 \mathrm{~V}$ and (b) hole sensitive at $+30 \mathrm{~V}$.

fields of different strengths and directions within the different areas of the detector. This will enhance the signal under external bias for one polarity and decrease it for the opposite one. The presence of these varying fields has been confirmed by signal pulses acquired without external bias supply. These $0 \mathrm{~V}$ data show signals of both polarities simultaneously depending on the detector position. The polarization and priming phenomena are discussed in more detail by Sellin et al. ${ }^{44}$ Due to the polarization, the electric field throughout the device is not known and probably not homogenous, which prevents a sensible evaluation of the voltage dependence of the $\mathrm{CCE}$ as a function of voltage for each pixel position in terms of the Hecht equation, which would yield a spatial $\mu \tau$ distribution. $^{45}$

Unfortunately, it is not clear yet how these variations in polarization are correlated to the spatial defect distribution within the sample; the data only suggest a systematic change along the growth direction, with clear changes across the nitrogen doped layers. It is known that nitrogen increases the diamond growth rate in polycrystalline $\mathrm{CVD}^{46}$ and therefore must influence some part of the synthesis mechanism, which could subsequently influence the defect distribution. Recent IBIC studies of polycrystalline diamond detectors at elevated temperatures up to $600 \mathrm{~K}$ suggest that space charge and thus polarization effects concentrated at grain boundaries are reduced at higher temperatures leading to a more homogenous detector response. $^{47}$

Although the performance of the sample studied in this work is expected to be affected by the nitrogen impurities which had been introduced on purpose, intrinsic space charge (internal fields ${ }^{19}$ ) and polarization effects ${ }^{48}$ have also been found by other groups in single crystal diamonds grown by the same company; but more recent results suggest that these internal space charges and/or polarization may not occur in more recently produced highest quality material, also synthesized by Element Six Ltd. ${ }^{49}$

The 10\%-90\% rise time image extracted from the digital IBIC data acquired simultaneously to the data shown in Fig. 8(b) at $+30 \mathrm{~V}$ is displayed in Fig. 9. It highlights that the pulse rise times are longer in two distinct areas. One of them, labeled $\mathrm{A}$, has very low $\mathrm{CCE}$ values, whereas in area $\mathrm{B}$, just above the HTHP substrate, CCEs are above $80 \%$, even at this low field strength. Induced charge pulses acquired within areas A and B at 296 and $270 \mathrm{~K}$ are shown in Figs. 10 and 11 , respectively. They demonstrate that the rise times of the 


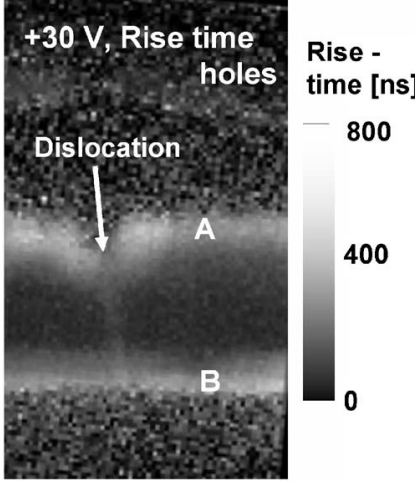

FIG. 9. 10\%-90\% rise time distribution of hole transport sensitive pulses acquired at $296 \mathrm{~K},+30 \mathrm{~V}$. The corresponding CCE image is shown in Fig. 8 (b). Pulse shapes extracted from areas A and B are shown in Figs. 10 and 11.

pulses originating in area A vary with applied bias voltage and are similar for the two temperatures. Thus, the slow charge transport in this area is caused by a reduced charge carrier velocity, hardly dependent on temperature. The reduction of hole velocity in this region is in agreement with the spatial distribution of polarization observed in this sample, i.e., reduced effective field strength.

In contrast, the pulses induced in area B shown in Fig. 11 display the characteristic combination of a fast component and a slow component, typical for delayed charge transport due to detrapping. The slow component has a clearly longer rise time at $270 \mathrm{~K}$ compared to $296 \mathrm{~K}$ and does not vary with the applied electric field. It is in agreement with the time characteristic of the trap level analyzed and discussed earlier. Our measurement shows that the responsible defect is only found very close to the substrate/CVD interface and could be caused by boron introduced during the initial growth stage into the CVD material from the substrate. The same reason might explain the observation of that trap in a

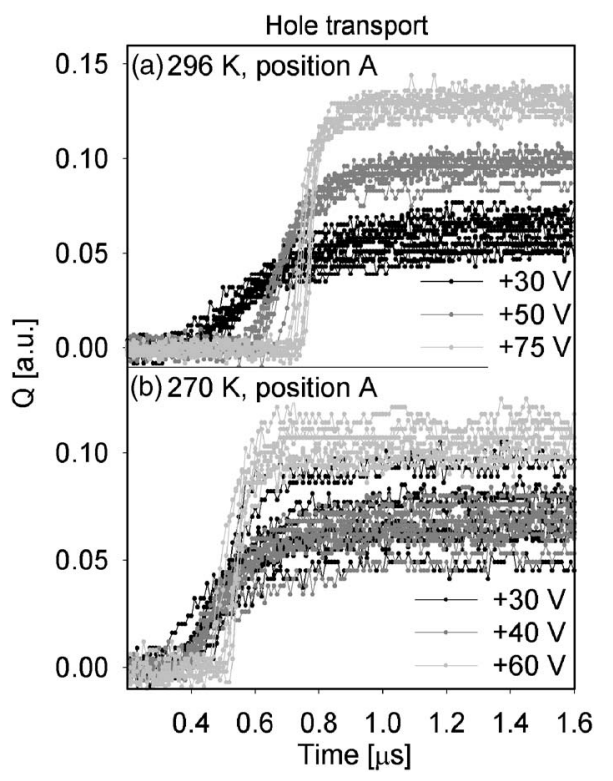

FIG. 10. Induced pulse shapes extracted from position A indicated in Fig. 9 at various bias voltages: (a) at $296 \mathrm{~K}$ and (b) at $270 \mathrm{~K}$.

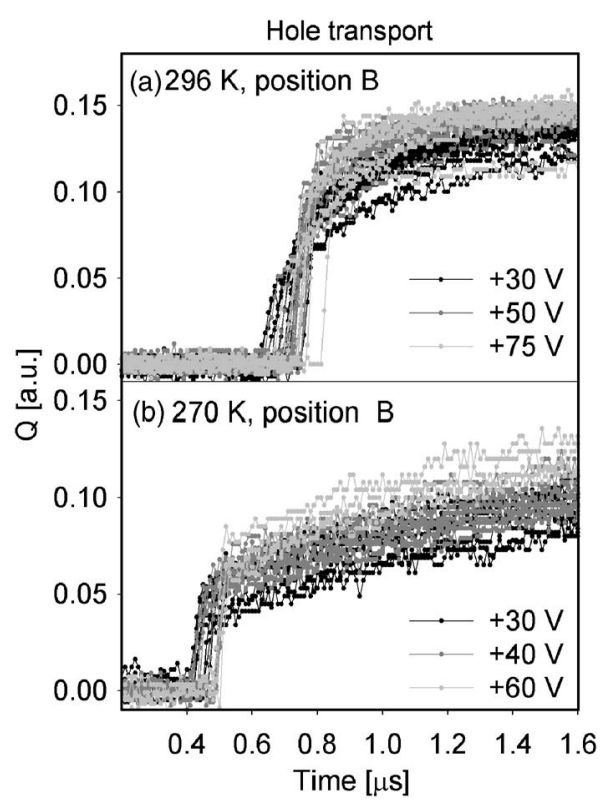

FIG. 11. Induced pulse shapes extracted from position B indicated in Fig. 9 at various bias voltages: (a) at $296 \mathrm{~K}$ and (b) at $270 \mathrm{~K}$.

thin $(80 \mu \mathrm{m})$ nominally undoped single crystal CVD diamond grown on an HTHP diamond substrate by Balducci et $a .^{25}$

\section{CONCLUSION}

The charge transport in a synthetic single crystal diamond containing areas of deliberately introduced nitrogen, grown by Element Six Ltd., has been studied. The $\mu \tau$ product for electrons and holes did not change significantly with temperature between 200 and $300 \mathrm{~K}$, which is in agreement with a very small increase of mobility within that temperature range. ${ }^{3}$

Polarization has been found in the device, and IBIC imaging revealed that the internal polarization field distribution seems to be related to the growth direction of the crystal. Furthermore, hole transport was strongly affected by incident dose rate changes due to priming.

We have confirmed the degradation of charge transport properties for electrons and holes related to nitrogen impurities. Additionally, a weak degradation due to the presence of dislocations was also found, which is in agreement with literature suggesting that nitrogen as well as dislocations can act as recombination centres ${ }^{40}$ and therefore reduce carrier lifetime.

Re-emission of holes out of shallow trap levels was observed in transient charge pulses. We presented a method to extract the detrapping time by fitting the slow component of these transient wave forms on a pulse by pulse basis. Analysis of these pulses as a function of temperature yields an activation energy of $0.29 \pm 0.02 \mathrm{eV}$, which indicates possibly a boron related hole trap.

Digital IBIC imaging was used to probe spatial variations in charge transport, and it has been shown that the effect of that shallow trap on the diamond detector signal is limited to an area very close to the HTHP substrate/CVD interface of the material. It was clearly distinguished from a 
reduction in charge carrier velocity due to polarization effects in other parts of the detector, which seemed to vary along the diamond growth direction of the CVD material.

\section{ACKNOWLEDGMENTS}

We are grateful to Element Six Ltd. for the supply of their material and for fruitful discussions especially with D. Twitchen and H. Murphy. We also thank R. W. Martin and P. R. Edwards at Strathclyde University, Glasgow (UK) for the cathodoluminescence measurements and G. Hill at the University of Sheffield (UK) for the preparation of the metallic contacts. This project has been funded by the EPSRC Grant No. GR/R34486/0.

${ }^{1}$ A. Mainwood, Semicond. Sci. Technol. 15, R55 (2000).

${ }^{2}$ P. Bergonzo, D. Tromson, and C. Mer, Semicond. Sci. Technol. 18, S105 (2003).

${ }^{3}$ C. E. Nebel and M. Stutzmann, in Electronic Materials Information Service, edited by M. H. Nazaré and A. J. Neves (INSPEC, London, 2001), Vol. 26, Chap. A1.6, p. 40.

${ }^{4}$ A. Mainwood, Diamond Relat. Mater. 7, 504 (1998).

${ }^{5}$ D. Meier et al., Nucl. Instrum. Methods Phys. Res. A 426, 173 (1999).

${ }^{6}$ A. Yacoot, M. Moore, and A. Makepiece, Phys. Med. Biol. 35, 1409 (1990).

${ }^{7}$ V. K. Liechtenstein et al., Nucl. Instrum. Methods Phys. Res. A 521, 203 (2004).

${ }^{8}$ J. Hammersberg, J. Isberg, E. Johansson, T. Lundström, O. Hjortestam, and H. Bernhoff, Diamond Relat. Mater. 10, 574 (2001).

${ }^{9}$ C. Manfredotti, F. Fizzotti, P. Polesollo, E. Vittone, and F. Wang, Phys. Status Solidi A 154, 327 (1994).

${ }^{10}$ S.-T. Lee, Z. Lin, and X. Jiang, Mater. Sci. Eng., R. 25, 123 (1999).

${ }^{11}$ J. Isberg, J. Hammersberg, E. Johansson, T. Wikström, D. J. Twitchen, A J. Whitehead, S. E. Coe, and G. A. Scarsbrook, Science 297, 1670 (2002).

${ }^{12}$ A. Tallaire, J. Achard, F. Silva, R. S. Sussmann, and A. Gicquel, Diamond Relat. Mater. 14, 249 (2005)

${ }^{13}$ T. Tanaka, J. H. Kaneko, Y. Kasugai, M. Katagiri, H. Takeuchi, T. Nishitani, and T. Iida, Diamond Relat. Mater. 14, 2031 (2005).

${ }^{14}$ C. Manfredotti, Diamond Relat. Mater. 14, 531 (2005).

${ }^{15}$ A. Bettiol, D. N. Jamieson, S. Prawer, and M. Allen, Nucl. Instrum. Methods Phys. Res. B 85, 775 (1994).

${ }^{16}$ A. Simon, P. J. Sellin, A. Lohstroh, and C. Jeynes, Nucl. Instrum. Methods Phys. Res. B 219-220, 1022 (2004).

${ }^{17}$ M. Marinelli, E. Milani, A. Paoletti, G. Pucella, A. Tucciarone, G. VeronaRinati, M. Angelone, and M. Pillon, Diamond Relat. Mater. 12, 1081 (2003).

${ }^{18}$ S. G. Wang, P. J. Sellin, and A. Lohstroh, Appl. Phys. Lett. 88, 023501 (2006).

${ }^{19}$ H. Pernegger et al., J. Appl. Phys. 97, 073704 (2005).

${ }^{20}$ M. Pomorski, E. Berdermann, M. Ciobanu, A. Martemyianov, P. Moritz, M. Rebisz, and B. Marczewska, Phys. Status Solidi A 202, 2199 (2005).

${ }^{21}$ G. F. Knoll, Radiation Detection and Measurement, 3rd ed. (Wiley, New
York, 1999).

${ }^{22}$ E. Souw and R. J. Meilunas, Nucl. Instrum. Methods Phys. Res. A 400, 69 (1997).

${ }^{23}$ C. Manfredotti, F. Fizzotti, P. Polesollo, and E. Vittone, Nucl. Instrum. Methods Phys. Res. A 426, 156 (1999)

${ }^{24}$ M. J. Guerrero, D. Tromson, M. Rebisz, C. Mer, B. Bazin, and P. Bergonzo, Diamond Relat. Mater. 13, 2046 (2004).

${ }^{25}$ A. Balducci, M. Marinelli, E. Milani, M. E. Morgada, G. Prestopinom, M. Scoccia, A. Tucciarone, and G. Verona-Rinati, Appl. Phys. Lett. 87, 222101 (2005).

${ }^{26}$ D. K. Schroder, Semiconductor Material and Device Characterisation (Wiley, New York, 1998).

${ }^{27}$ Courtesy of Element Six, Ltd.

${ }^{28}$ T. Tachibana, B. E. Williams, and J. T. Glass, Phys. Rev. B 45, 11975 (1992).

${ }^{29}$ A. Simon et al., Nucl. Instrum. Methods Phys. Res. B 219-220, 405 (2004).

${ }^{30}$ C. Canali, E. Gatti, F. Kozlov, F. Manfredi, C. Manfredotti, F. Nava, and A. Quirini, Nucl. Instrum. Methods 160, 73 (1979).

${ }^{31}$ http://www.srim.org; J. P. Biersack and L. Haggmark, Nucl. Instrum. Methods 174, 257 (1980).

${ }^{32}$ S. G. Wang, P. J. Sellin, and A. Lohstroh, Carbon 43, 3167 (2005).

${ }^{33}$ P. J. Sellin, A. Lohstroh, and A. Simon, Nucl. Instrum. Methods Phys. Res. A 521, 600 (2004).

${ }^{34}$ A. Lohstroh, P. J. Sellin, S. G. Wang, A. W. Davies, J. Parkin, R. W. Martin, and P. R. Edwards, Appl. Phys. Lett. 90, 102111 (2007).

${ }^{35}$ K. Iakoubovskii, G. J. Adriaenssens, M. Nesladek, and L. Stals, Diamond Relat. Mater. 8, 717 (1999).

${ }^{36}$ J. Ruan, K. Kobashi, and W. J. Choyke, Appl. Phys. Lett. 60, 3138 (1992).

${ }^{37}$ Y. Tanimura, J. Kaneko, M. Katagiri, Y. Ikeda, T. Nishitani, H. Takeuchi, and T. Iida, Nucl. Instrum. Methods Phys. Res. A 443, 325 (2002).

${ }^{38}$ J. W. Glesener, Appl. Phys. Lett. 63, 767 (1993).

${ }^{39}$ H. Sato, H. Tomokage, H. Kiyota, and T. Ando, Diamond Relat. Mater. 7, 1167 (1998).

${ }^{40}$ L. S. Pan, D. R. Kania, P. Pianetta, J. W. Ager, III, M. I. Landstrass, and S. Han, J. Appl. Phys. 73, 2888 (1993).

${ }^{41}$ P. Bergonzo, R. Barrett, O. Hainaut, D. Tromson, C. Mer, and B. Guizard, Diamond Relat. Mater. 11, 418 (2002).

${ }^{42}$ M. Marinelli, E. Milani, A. Paoletti, A. Tucciarone, and G. Verona-Rinati, Appl. Phys. Lett. 75, 3216 (1999).

${ }^{43}$ M. Donato et al., Diamond Relat. Mater. 10, 1788 (2000).

${ }^{44}$ P. J. Sellin, A. Lohstroh, A. W. Davies, A. Galbiati, J. Parkin, S. G. Wang, and A. Simon, Nucl. Instrum. Methods Phys. Res. B (submitted).

${ }^{45}$ A. Lohstroh, P. J. Sellin, and A. Simon, J. Phys.: Condens. Matter 16, S67 (2004).

${ }^{46}$ J. Achard, A. Tallaire, R. Sussmann, R. Silva, and A. Gicquel, J. Cryst. Growth 284, 396 (2005).

${ }^{47}$ S. M. Hearne, E. Trajkov, D. N. Jamieson, J. E. Butler, and S. Prawer, J. Appl. Phys. 99, 113703 (2006).

${ }^{48}$ G. J. Schmidt, J. A. Koch, R. A. Lerche, and M. J. Moran, Nucl. Instrum. Methods Phys. Res. A 527, 554 (2004).

${ }^{49}$ NoRHDia Collaboration, M. Pomorski, E. Berdermann, A. Caragheorgheopol, M. Ciobanu, M. Kiš, A. Martemiyanov, C. Nebel, and P. Moritz, Phys. Status Solidi A 203, 3152 (2006). 